\title{
Colorimetric Sensitivity of Gold Nanoparticles: Minimizing Interparticular Repulsion as a General Approach
}

\author{
Sung-Hsun Wu, ${ }^{\dagger}$ Yi-Shan $\mathbf{W u},{ }^{\dagger}$ and Chun-hsien Chen*,‡ \\ Department of Chemistry, National Tsing Hua University, Hsinchu, Taiwan 30013, and Department of Chemistry, \\ National Taiwan University, Taipei, Taiwan 10617
}

GNPs (gold nanoparticles) as an eye-catching sensor rely on the high extinction coefficients and the shift of the surface plasmon band which signals the disperse-toaggregate transformation. The selectivity of the sensors is dictated by the surface functionality whose density presumably has a positive correlation with the sensitivity toward the targeted analyte. To improve the analytical performance, most efforts in this research field focus on the design and synthesis of the sensing elements as well as on the increase in density on GNPs. Proposed here is an alternative rationale that the further improvement of the GNP sensitivity can be achieved by minimizing the electrostatic repulsion and hence the energy barrier for the recognition event to take place. Our model system begins with thioctic acid-stabilized GNPs which are subsequently modified with 15 -crown-5 ether for the recognition toward $\mathrm{K}^{+}$. For a given coverage of 15 -crown- 5 ether, the limits of detection (LODs) can be improved by more than 3 orders of magnitude via adjusting the solution $\mathrm{pH}$ and ionic strength which we suggest a general guideline for the optimization of a new GNP sensing scheme. Following this guideline, satisfactory performance with LODs at the micromolar level can be systematically and efficiently found for GNPs with a range of 15-crown-5 ether coverage.

Gold nanoparticles (GNPs) have received great attention in the development of visual sensing schemes because of their high extinction coefficients and strongly proximity-dependent surface plasmon band which render the distinct color transformation in responding to the recognition events associated with the dispersion-aggregation transition of GNPs. ${ }^{1-3}$ Such analyte-triggered signaling of the GNP colors is dictated by interactions that, although oversimplified, can always be broken down into interparticular attraction and repulsion. The attractive interactions have been the center of focus to improve the sensing performance. Successfully engineered strategies reported in literature are

\footnotetext{
* To whom correspondence should be addressed. Phone: +88623366 4191. Fax: +886 22363 6359. E-mail: chhchen@ntu.edu.tw.

${ }^{\dagger}$ National Tsing Hua University.

* National Taiwan University.

(1) Daniel, M.-C.; Astruc, D. Chem. Rev. 2004, 104, 293-346.

(2) Rosi, N. L.; Mirkin, C. A. Chem. Rev. 2005, 105, 1547-1562.

(3) Drechsler, U.; Erdogan, B.; Rotello, V. M. Chem.-Eur. J. 2004, 10, 55705579.
}

examples such as (1) maximizing the amount of the recognition functionality on GNPs to increase the sensitivity, ${ }^{4-8}$ (2) tailoring the sensing moieties to pursue the affinity and specificity toward analytes, ${ }^{2-6,9-37}$ and (3) preorganizing the sensing group to

(4) Zheng, M.; Huang, X. J. Am. Chem. Soc. 2004, 126, 12047-12054.

(5) Huang, C.-C.; Huang, Y.-F.; Cao, Z.; Tan, W.; Chang, H.-T. Anal. Chem. 2005, 77, 5735-5741.

(6) Thanh, N. T. K.; Rosenzweig, Z. Anal. Chem. 2002, 74, 1624-1628. To the best of our knowledge, thus far this is the only report addressing this issue. The GNPs were coated by protein A whose surface charge and thus the aggregation were tuned by solution acidity.

(7) Takae, S.; Akiyama, Y.; Otsuka, H.; Nakamura, T.; Nagasaki, Y.; Kataoka, K. Biomacromolecules 2005, 6, 818-824.

(8) Hurst, S. J.; Lytton-Jean, A. K. R.; Mirkin, C. A. Anal. Chem. 2006, 78, 8313-8318.

(9) Han, M. S.; Lytton-Jean, A. K. R.; Mirkin, C. A. J. Am. Chem. Soc. 2006, 128, 4954-4955.

(10) Si, S.; Kotal, A.; Mandal, T. K. J. Phys. Chem. C 2007, 111, 1248-1255.

(11) Huang, C.-C.; Chang, H.-T. Chem. Commun. 2007, 1215-1217.

(12) Watanabe, S.; Seguchi, H.; Yoshida, K.; Kifune, K.; Tadaki, T.; Shiozaki, H. Tetrahedron Lett. 2005, 46, 8827-8829.

(13) Ma, Y.; Li, N.; Yang, C.; Yang, X. Colloid Surf. A: Physicochem. Eng. Asp. 2005, 269, 1-6.

(14) Ma, Y.; Li, N.; Yang, C.; Yang, X. Anal. Bioanal. Chem. 2005, 382, 10441048.

(15) Lu, C.; Zu, Y.; Yam, V. W.-W. Anal. Chem. 2007, 79, 666-672.

(16) Schofield, C. L.; Field, R. A.; Russell, D. A. Anal. Chem. 2007, 79, 13561361.

(17) Schofield, C. L.; Haines, A. H.; Field, R. A.; Russell, D. A. Langmuir 2006, 22, 6707-6711.

(18) Reynolds, A. J.; Haines, A. H.; Russell, D. A. Langmuir 2006, 22, 11561163.

(19) Hone, D. C.; Haines, A. H.; Russell, D. A. Langmuir 2003, 19, 7141-7144.

(20) Liu, J.; Lu, Y. Anal. Chem. 2004, 76, 1627-1632.

(21) Liu, J.; Lu, Y. J. Am. Chem. Soc. 2003, 125, 6642-6643.

(22) Obare, S. O.; Hollowell, R. E.; Murphy, C. J. Langmuir 2002, 18, 1040710410.

(23) Wang, Z.; Levy, R.; Fernig, D. G.; Brust, M. Bioconjugate Chem. 2005, 16, 497-500.

(24) Lin, S.-Y.; Liu, S.-W.; Lin, C.-M.; Chen, C.-h. Anal. Chem. 2002, 74, 330335.

(25) Lin, S.-Y.; Wu, S.-H.; Chen, C.-h. Angew. Chem., Int. Ed. 2006, 45, 49484951.

(26) Yang, W.; Gooding, J. J.; He, Z.; Li, Q.; Chen, G. J. Nanosci. Nanotechnol. 2007, 7, 712-716.

(27) Sato, K.; Hosokawa, K.; Maeda, M. Anal. Sci. 2007, 23, 17-20.

(28) Okubo, K.; Shimada, T.; Shimizu, T.; Uehara, N. Anal. Sci. 2007, 23, 8590.

(29) Kubo, Y.; Uchida, S.; Kemmochi, Y.; Okubo, T. Tetrahedron Lett. 2005, 46, 4369-4372.

(30) Aslan, K.; Zhang, J.; Lakowicz, J. R.; Geddes, C. D. J. Fluoresc. 2004, 14, 391-400.

(31) Aslan, K.; Lakowicz, J. R.; Geddes, C. D. Anal. Chem. 2005, 77, 20072014.

(32) Aslan, K.; Lakowicz, J. R.; Geddes, C. D. Anal. Chim. Acta 2004, 517, 139-144. Published on Web 07/29/2008 
accelerate the complexation with analytes. ${ }^{37-41}$ On the other hand, the repulsive force is considered to play a passive role that functions limitedly to stabilize GNPs, rather than to enhance the colorimetric response. In the field of colloidal science, the stability of dispersive colloids is commonly rationalized by the DLVO theory (after Derjaguin, Landau, Vervey, and Overbeek). ${ }^{42}$ This theory quantitatively explains the interactions between particles by the equation, $V_{\mathrm{T}}=V_{\mathrm{vdw}}+V_{\text {elec}}$, with the potential energy, $V_{\mathrm{T}}$, being the sum of the energies from the van der Waals attractions, $V_{\text {vdw }}$, and the electrostatic repulsions, $V_{\text {elec }}$. The contributions of $V_{\text {vdw }}$ and $V_{\text {elec }}$ to $V_{\mathrm{T}}$ generally vary as the inverse sixth power and the inverse of the separation between the particles, respectively. In terms of distance dependence, $V_{\mathrm{vdw}}$ is a short-range interaction and $V_{\text {elec }}$ is a long-range one. The recognition events generally require the sensing moiety to host the guest analyte via multiple affinity sites, and thus the recognition events can be considered as contactlike interactions that a smaller $V_{\text {elec }}$ can reduce the energy barrier for the sensing reaction to take place.

Much progress has been made in recent years for water-soluble GNPs that feature a variety of thiolated monolayers. ${ }^{3-24,43-57}$ Although the buffer compositions ${ }^{11}$ and ionic strength ${ }^{5}$ have been mentioned in the consideration of interparticlar interactions, how the sensing performance can be optimized by tuning the electrostatic repulsion has not yet been subjected to detailed examina-

(33) Storhoff, J. J.; Lucas, A. D.; Garimella, V.; Bao, Y. P.; Muller, U. R. Nat. Biotechnol. 2004, 22, 883-887.

(34) Daniel, M.-C.; Ruiz, J.; Nlate, S.; Blais, J.-C.; Astruc, D. J. Am. Chem. Soc. 2003, 125, 2617-2628.

(35) Labande, A.; Ruiz, J.; Astruc, D. J. Am. Chem. Soc. 2002, 124, 1782-1789.

(36) Boal, A. K.; Rotello, V. M. J. Am. Chem. Soc. 2002, 124, 5019-5024.

(37) Descalzo, A. B.; Martinez-Manez, R.; Sancenon, R.; Hoffmann, K.; Rurack, K. Angew. Chem., Int. Ed. 2006, 45, 5924-5948.

(38) Lin, S.-Y.; Chen, C.-h.; Lin, M.-C.; Hsu, H.-F. Anal. Chem. 2005, 77, 48214828.

(39) Arduini, A.; Demuru, D.; Pochini, A.; Secchi, A. Chem. Commun. 2005, $645-647$.

(40) Tshikhudo, T. R.; Demuru, D.; Wang, Z.; Brust, M.; Secchi, A.; Arduini, A.; Pochini, A. Angew. Chem., Int. Ed. 2005, 44, 2913-2916.

(41) Boal, A. K.; Rotello, V. M. J. Am. Chem. Soc. 2000, 122, 734-735.

(42) Hunter, R. J. Foundations of Colloid Science, 2nd ed.; Oxford University: New York, 2001.

(43) Simard, J.; Briggs, C.; Boal, A. K.; Rotello, V. M. Chem. Commun. 2000, 1943-1944.

(44) Love, J. C.; Estroff, L. A.; Kriebel, J. K.; Nuzzo, R. G.; Whitesides, G. M. Chem. Rev. 2005, 105, 1103-1169.

(45) Mangeney, C.; Ferrage, F.; Aujard, I.; Marchi-Artzner, V.; Jullien, L.; Ouari, O.; Rekai, E. D.; Laschewsky, A.; Virholm, I.; Sadowski, J. W. J. Am. Chem. Soc. 2002, 124, 5811-5821.

(46) Aslan, K.; Perez-Luna, V. H. Langmuir 2002, 18, 6059-6065.

(47) Stoeva, S. I.; Smetana, A. B.; Sorensen, C. M.; Klabunde, K. J. J. Colloid Interface Sci. 2007, 309, 94-98.

(48) Grace, A. N.; Pandian, K. Colloid Surf. A: Physicochem. Eng. Asp. 2007, 297, 63-70.

(49) Weisbecker, C. S.; Merritt, M. V.; Whitesides, G. M. Langmuir 1996, 12, 3763-3772.

(50) Lin, S.-Y.; Tsai, Y.-T.; Chen, C.-C.; Lin, C.-M.; Chen, C.-h. J. Phys. Chem. B 2004, 108, 2134-2139.

(51) Lowe, A. B.; Sumerlin, B. S.; Donovan, M. S.; McCormick, C. L. J. Am. Chem. Soc. 2002, 124, 11562-11563.

(52) Huang, C.-C.; Chang, H.-T. Anal. Chem. 2006, 78, 8332-8338.

(53) Qi, Z.-m.; Zhou, H.-s.; Matsuda, N.; Honma, I.; Shimada, K.; Takatsu, A.; Kato, K. J. Phys. Chem. B 2004, 108, 7006-7011.

(54) Abad, J. M.; Mertens, S. F. L.; Pita, M.; Fernandez, V. M.; Schiffrin, D. J. J. Am. Chem. Soc. 2005, 127, 5689-5694.

(55) Chen, S.-J.; Chang, H.-T. Anal. Chem. 2004, 76, 3727-3734.

(56) Chen, Y.-M.; Yu, C.J.; Cheng, T.-L.; Tseng, W.-L. Langmuir 2008, 24, 36543660.

(57) Shipway, A. N.; Lahav, M.; Gabai, R.; Willner, I. Langmuir 2000, 16, 87898795. tion. ${ }^{6}$ Herein, the model system is a potassium sensor. Potassium is one of the vital electrolytes in the human body. The urine potassium falls in the range of $25 \sim 125 \mathrm{mEq} /$ day which varies with diet. ${ }^{58,59}$ The urine volumes for adult males and females are, respectively, 800 1800 mL/day and 600 1600 mL/day, which vary with water intake and other factors. An abnormally high level of urine potassium indicates aldosteronism, alkalosis, renal tubular acidosis, or excess potassium intake while a low level suggests acute renal failure, diarrhea, or hypokalemia. ${ }^{58,59}$ The potassium sensor is denoted $15 \mathrm{c} 5-\mathrm{C}_{4}$-/TA-GNPs that the GNPs are bifunctinoalized by thioctic acid (TA, I) and 2-(4-mercaptobutyloxy)methyl-15-crown-5 ether (15-crown-5- $\mathrm{CH}_{2} \mathrm{O}\left(\mathrm{CH}_{2}\right)_{4} \mathrm{SH}$ or $15 \mathrm{c} 5-\mathrm{C}_{4}-\mathrm{SH}$, II). The presence of $\mathrm{K}^{+}$induces a 2-to- 1 sandwich complexation of 15 -crown-5 to $\mathrm{K}^{+}, 24,38,60-66$ leading to the aggregation of GNPs and a red-to-blue transformation. ${ }^{24,38}$ Employment of TA to modify GNPs has the advantages of stability that arises from the bidentate $\mathrm{S}-\mathrm{Au}$ ligation and from the negative charge carried at the carboxylate. ${ }^{50}$ In the following, the electrostatic repulsion between $15 \mathrm{c} 5-\mathrm{C}_{4}$-/TA-GNPs is tuned by adjusting $\mathrm{pH}$ and ionic strength. The limits of detection (LODs) are thoroughly examined for a range of experimental conditions and are utilized to evaluate the effect of minimizing interparticular repulsion. This study demonstrates a guideline for the condition optimization when a novel GNP sensing scheme is under development.
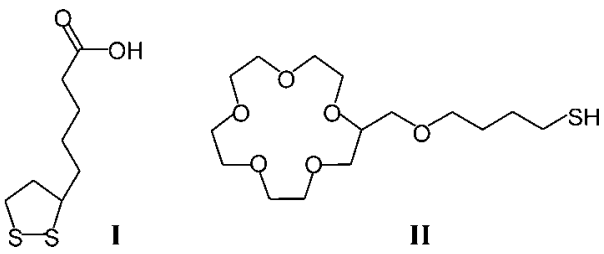

\section{EXPERIMENTAL SECTION}

General Information. All chemicals were reagent grade. Buffer solutions were $10.0 \mathrm{mM}$ MES (2-( $N$-morpholino)ethanesulfonic acid, Sigma) adjusted to desired $\mathrm{pH}$ and ionic strength with 0.5 $\mathrm{N} \mathrm{NaOH}$ and $0.5 \mathrm{~N} \mathrm{NaCl}$, respectively. Synthesis of 2-(4mercaptobutyloxy) methyl-15-crown-5 ether (15c5- $\left.\mathrm{C}_{4}-\mathrm{SH}\right)$ was described elsewhere. ${ }^{24,67}$ Colloidal gold was prepared by the protocol of Natan and co-workers. ${ }^{68}$ The average diameter of $19.6 \pm 3.1$ $\mathrm{nm}$ was measured by transmission electron microscopy (TEM, Hitachi, H-7500) and determined with Scion Image Beta Release 2 (available at www.scioncorp.com). The bifunctionalized 15c5-

(58) Tietz, N. W.; Logan, N. M. In Fundamentals of Clinical Chemistry, 3rd ed.; Tietz, N. W., Ed.; Saunders: Philadelphia, PA, 1987; pp 616-618, 963.

(59) Seldin, D. W.; Giebisch, G. The Regulation of Potassium Balance; Raven Press: New York, 1989.

(60) Christensen, J. J.; Hill, J. O.; Izatt, R. M. Science 1971, 174, 459-467.

(61) Xia, W.-S.; Schmehl, R. H.; Li, C.-J. J. Am. Chem. Soc. 1999, 121, 55995560 .

(62) Yamauchi, A.; Hayashita, T.; Nishizawa, S.; Watanabe, M.; Teramae, N. J. Am. Chem. Soc. 1999, 121, 2319-2320.

(63) Kim, J.; McQuade, D. T.; McHugh, S. K.; Swager, T. M. Angew. Chem., Int. Ed. 2000, 39, 3868-3872.

(64) Chen, C.-Y.; Cheng, C.-T.; Lai, C.-W.; Wu, P.-W.; Wu, K.-C.; Chou, P.-T.; Chou, Y.-H.; Chiu, H.-T. Chem. Commun. 2006, 263-265.

(65) Nakashima, H.; Furukawa, K.; Kashimura, Y.; Torimitsu, K. Chem. Commun. 2007, 1080-1082.

(66) Pompano, R. R.; Wortley, P. G.; Moatz, L. M.; Tognarelli, D. J.; Kittredge, K. W.; Leopold, M. C. Thin Solid Films 2006, 510, 311-319.

(67) Flink, S.; Boukamp, B. A.; van den Berg, A.; van Veggel, F. C. J. M.; Reinhoudt, D. N. J. Am. Chem. Soc. 1998, 120, 4652-4657.

(68) Grabar, K. C.; Freeman, R. G.; Hommer, M. B.; Natan, M. J. Anal. Chem. 1995, 67, 735-743. 

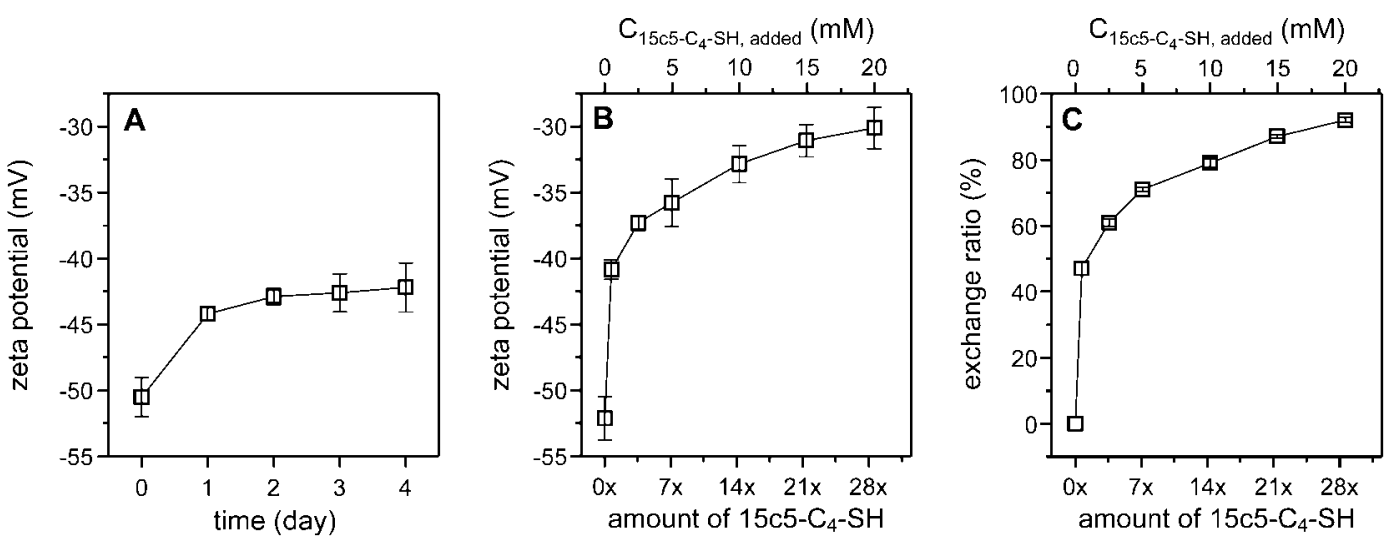

Figure 1. $\xi$ potentials and surface compositions of the colloidal gold. (A) $\zeta$ monitored as a function of stirring time for $0.64 \times$ GNPs. (B) $\zeta$ and (C) exchange ratio for GNPs prepared by 4 days of stirring with the indicated amount of $15 \mathrm{c} 5-\mathrm{C}_{4}-\mathrm{SH}$. The exchange ratio was determined by the oxygen to sulfur abundance ratios from XPS measurements (see text). The values on the bottom abscissa describe the mole ratios of the introduced $15 \mathrm{c5}-\mathrm{C}_{4}-\mathrm{SH}$ to those estimated from a full monolayer on GNPs (see Experimental Section). The solutions were prepared by filling Millipore-Q water to purified TA-GNPs without introducing electrolytes or adjusting the solution pH deliberately. A $1.00 \mathrm{~mL}$ aliquot of $15 \mathrm{c} 5-\mathrm{C}_{4}-\mathrm{SH}$ was introduced to $40.0 \mathrm{~mL}$ of TA-GNPs $\left(3.00 \mathrm{AU}\right.$ at $\left.\lambda_{523 \mathrm{~nm}}\right){ }^{46,50,76-78}$ The upper abscissa shows the concentration of $15 \mathrm{c5}-\mathrm{C}_{4}-\mathrm{SH}$ in the 1.00 $\mathrm{mL}$ aliquot. The GNPs used to determine the compositions in part $\mathrm{C}$ by XPS measurements were from the corresponding batch of part $\mathrm{B}$.

$\mathrm{C}_{4}$-/TA-GNPs were prepared by the previous protocol..$^{50}$ Instruments employed to carry out the measurements of weight percentage of the organic monolayer on GNPs, $\zeta$ potentials, absorbance of the SP band, and semiquantification of oxygen to sulfur abundance at the organic monolayer of the GNPs were, respectively, thermogravimetric analyzer (HI-RES TGA 2950, TA Instruments), Zetasizer (Nano ZS, Malvern), UV-visible spectrophotometer (UV 300, Unicam), and XPS (ESCA PHI 1600, Physical Electronics).

Amount of 15c5- $\mathrm{C}_{4}$-SH for Ligand-Exchange Modification. The surface coverage of the crown ether moiety was adjusted by the amounts of $15 \mathrm{c} 5-\mathrm{C}_{4}-\mathrm{SH}$ introduced to and stirred with the solution of TA-GNPs. This approach was based on the ligandexchange methodology that the mole ratio of in-coming ligand against those on GNPs decides the final surface composition. ${ }^{69-71}$ To select a range of mole ratios for functionalization, we began with estimating the total number of the thiol headgroup on GNPs per $40.0 \mathrm{~mL}$ batch whose intensity of the SP band was fixed at 3.00 AU (without baseline correction). The weight of GNPs was estimated $0.30 \mathrm{mg} / \mathrm{mL}$ (Figure S2, the Supporting Information). The weight and surface area per gold core were approximated by the averaged particle diameter $(19.6 \pm 3.1 \mathrm{~nm})$ from TEM electromicrographs and by assuming $19.3 \mathrm{~g} / \mathrm{cm}^{3}$ in density and perfect spherical particles in shape. The overall weight of gold cores was deduced from the $2.5 \%$ weight loss of TA molecules from GNPs (Figure S3, the Supporting Information) measured at elevating temperatures $\left(200-400{ }^{\circ} \mathrm{C}\right)$. Accordingly, the number and surface area of the $40.0 \mathrm{~mL}$ GNP solution were, respectively, $\sim 7.38 \times 10^{13}$ particles and $8.86 \times 10^{16} \mathrm{~nm}^{2}$, leading to a maximum of $0.7 \mu \mathrm{mol}$ of thiol headgroup based on $0.216 \mathrm{~nm}^{2}$ per thiol for the close-packed arrangement. ${ }^{72}$ We therefore decided to add to the $40.0 \mathrm{~mL}$ of TA-GNPs the amounts of $0.50,2.50,5.00,10.0$, 15.0 , and $20.0 \mu \mathrm{mol}$ of $15 \mathrm{c} 5-\mathrm{C}_{4}-\mathrm{SH}(1.00 \mathrm{~mL})$, roughly $0.70,3.6$, $7.1,14.3,21.4$, and 28.6 times the maximum mole of thiol legs on GNPs, respectively. In the following manuscript, the mole ratio is utilized to denote how the nanoparticles were functionalized. For examples, $21.4 \times$ represents $15 \mathrm{c} 5-\mathrm{C}_{4}-\mathrm{TA}$-GNPs prepared by mixing $15.0 \mu \mathrm{mol}$ of $15 \mathrm{c}^{2}-\mathrm{C}_{4}$-SH with $40.0 \mathrm{~mL}$ of TA-GNPs $(3.00$ $\mathrm{AU}$ at $\lambda_{\max }$.
Preparation and Analysis of Human Urine Samples. Urine samples were collected from a volunteer and were not subjected to pretreatment other than dilution by the MES buffer that was also used in the preparation of GNPs solutions. Unless specifically stated, UV-vis spectra were acquired 3 min after introducing a $0.10 \mathrm{~mL}$ aliquot of the sample into $1.9 \mathrm{~mL}$ of GNP solutions. The calibration curves were obtained by correlating the concentration of analytes to the decrease in peak intensity of the dispersive SP band.

\section{RESULTS AND DISCUSSION}

Modification Time for the Preparation of 15c5-C $\mathrm{C}_{4} /$ /TA-GNPs. The sensitivity of GNPs toward $\mathrm{K}^{+}$-sensing is presumably decided by the surface composition; specifically, crown ether and carboxylate accounted for the recognition and the electrostatic stabilization, respectively. To yield a meaningful analysis on the sensing performance, it is essential to have a protocol for the preparation of GNPs whose number of ligands is controllable and reproducible to some extent.

In this study, IR spectra of $15 \mathrm{c} 5-\mathrm{C}_{4}-/ \mathrm{TA}-\mathrm{GNPs}$ were used to examine the degree of modification. The peak intensities for the characteristic vibrational modes of crown ether and carboxylate were strongly associated with the amount of $15 \mathrm{c} 5-\mathrm{C}_{4}-\mathrm{SH}$ introduced for functionalization and with the duration of the mixing time (see Figure S1 in Supporting Information). However, the information deduced from IR peak intensity was only qualitative and not in real time. Alternatively, the dynamic exchange of TA by the incoming $15 \mathrm{c}^{2}-\mathrm{C}_{4}-\mathrm{SH}$ was monitored by the $\zeta$ potential, the electrostatic potential at the surface of shear near the GNPs. ${ }^{73}$ Panel A of Figure 1 shows that, upon introducing $0.45 \mu \mathrm{mol}$ of $15 \mathrm{c} 5-\mathrm{C}_{4}-\mathrm{SH}$ into $40.0 \mathrm{~mL}$ of TA-GNPs, $|\zeta|$ decreased due to the displacement of the negatively charged TA by the neutral $15 \mathrm{c} 5$ -

(69) Kassam, A.; Bremner, G.; Clark, B.; Ulibarri, G.; Lennox, R. B. J. Am. Chem. Soc. 2006, 128, 3476-3477.

(70) Hostetler, M. J.; Templeton, A. C.; Murray, R. W. Langmuir 1999, 15, 3782-3789.

(71) Song, Y.; Murray, R. W. J. Am. Chem. Soc. 2002, 124, 7096-7102.

(72) Ulman, A. Chem. Rev. 1996, 96, 1533-1554.

(73) Hunter, R. J. Zeta Potential in Colloid Science; Academic Press: New York, 1981. 
Table 1. Numbers of Crown Thiols per GNP Estimated by XPS Measurements

\begin{tabular}{|c|c|c|c|c|}
\hline \multirow[b]{2}{*}{$15 \mathrm{c} 5-\mathrm{C}_{4}-/ \mathrm{TA}-\mathrm{GNPs}^{a}$} & \multicolumn{2}{|c|}{ binding energy (eV) } & 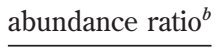 & \multirow[b]{2}{*}{ exchange ratio $^{c, d}(\%)$} \\
\hline & $\mathrm{S}_{2 \mathrm{p}_{3 / 2}}$ & $\mathrm{O}_{1 \mathrm{~s}}$ & $\mathrm{O} / \mathrm{S}$ & \\
\hline $0.7 \times$ & 162.8 & 532.6 & 3.35 & $47 \pm 2(64)^{d}$ \\
\hline $3.6 \times$ & 162.4 & 532.6 & 4.06 & $61 \pm 1(76)$ \\
\hline $7.1 \times$ & 162.6 & 533.8 & 4.55 & $71 \pm 1(83)$ \\
\hline $14.3 \times$ & 162.4 & 533.6 & 5.00 & $79 \pm 1(89)$ \\
\hline $21.4 \times$ & 162.4 & 533.6 & 5.33 & $87 \pm 1(93)$ \\
\hline $28.6 \times$ & 162.4 & 532.2 & 5.62 & $92 \pm 1(96)$ \\
\hline
\end{tabular}

${ }^{a}$ GNPs were centrifuged twice and drop-cast on thermally evaporated gold substrate. ${ }^{b}$ Calculations of the abundance ratio were based on peak area multiplied by the corresponding atomic sensitivity factor. ${ }^{c}$ The exchange ratio roughly represents the fraction of TA exchanged in the second step. The ratio is calculated from the abundance ratio (XPS) and by the number of crown thiol over the total number of thiol-legs where crown thiol and TA have one and two S-Au bonds per molecule, respectively. Specifically, the fraction and the abundance ratio should satisfy the fact that there are two sulfur atoms and two oxygen atoms per TA molecule, one sulfur atom, and six oxygen atoms per 15 -crown-5 thiol. The numbers of organic molecules per GNP were estimated by the following procedures. The weight ratio of $2.5 \%$ for the organic layer per GNP was measured by monitoring the weight loss of GNPs from 110 to $500^{\circ} \mathrm{C}$. The average diameter of $19.6 \pm 3.1 \mathrm{~nm}$ for GNPs was used to calculate the nominal mass of the gold core. Therefore, the numbers of crown thiols per GNPs were estimated from the weight fraction of organic layer (by TGA), the ratio of crown thiols over the total thiol (by XPS), and molecular mass. ${ }^{d}$ Reported in the parentheses are the percentages of the number of crown thiol over the total number of molecules on GNPs.

$\mathrm{C}_{4}$ - $\mathrm{SH}$ and thus the loss of surface charge. The place-exchange reaction reached equilibrium in less than 2 days. The profile revealed a relatively fast reaction at the initial stage followed by a slow one after 1 day, analogous to that of citrate-GNPs exchanged by thioctic acid in aqueous solution ${ }^{50}$ or that of decanethiol-protected gold clusters exchanged by dodecanethiol in organic phase. ${ }^{69,74,75}$ To obtain GNPs with a reasonably reproducible composition, the duration of mixing $15 \mathrm{c} 5-\mathrm{C}_{4}$ - $\mathrm{SH}$ with TA-GNP sol was decided 4 days or longer when the steady state equilibrium of the place-exchange reaction was attained.

For $15 \mathrm{c} 5-\mathrm{C}_{4}-/ \mathrm{TA}-\mathrm{GNPs}$ already at the steady state, another addition of $15 \mathrm{c} 5-\mathrm{C}_{4}-\mathrm{SH}$ led to a further decrease in $|\xi|$ (data not shown), suggesting that the extent of the place-exchange reaction was determined by the molar ratio of incoming to outgoing ligand. Accordingly, the surface composition of $15 \mathrm{c} 5-\mathrm{C}_{4}-/ \mathrm{TA}-\mathrm{GNPs}$ was controllable. Panel B of Figure 1 demonstrates that $|\xi|$ was a function of the introduced amount of $15 \mathrm{c} 5-\mathrm{C}_{4}-\mathrm{SH}$. $|\xi|$ of $28.6 \times \mathrm{GNPs}$ was $30.1 \pm 1.6 \mathrm{mV}$, approaching the limiting value of $\sim 25 \mathrm{mV}$ for nanoparticles being stable exclusively by electrostatic repulsion. ${ }^{79}$ The attempt to prepare 38.6× GNPs was failed due to the development of unstable purplish flocculation. Therefore, the mole ratios examined in this study ranged from $0.70 \times$ to $28.6 \times$ GNPs.

Panel C of Figure 1 quantitatively describes the exchange ratio. The GNPs prepared for Figure 1B were subsequently subjected to the measurements of X-ray photoelectron spectroscopy (XPS) which conferred the abundance ratio of oxygen to sulfur atoms. From the abundance ratio and the numbers of sulfur and oxygen atoms per molecule (two sulfur and two oxygen atoms per TA; one sulfur and six oxygen atoms per $15 \mathrm{c}^{-}-\mathrm{C}_{4}-\mathrm{SH}$ ), the percentages of the crown thiols to total number of thiol legs per GNP were in the range of $47-92 \%$ (Table 1). The traces in panels B and C are quite similar, suggesting that the measurements of $\zeta$ for the GNPs

(74) Donkers, R. L.; Song, Y.; Murray, R. W. Langmuir 2004, 20, 4703-4707.

(75) Guo, R.; Song, Y.; Wang, G.; Murray, R. W. J. Am. Chem. Soc. 2005, 127, $2752-2757$.

(76) $\lambda_{\max }$ of $523 \sim 525 \mathrm{~nm}$ are reported in this manuscript. The variation in the $\lambda_{\max }$ arises from the dielectrics of the organic monolayers rather than from the size of GNPs. See ref 4677 , and 78 .

(77) Mulvaney, P. Langmuir 1996, 12, 788-800.

(78) Nath, N.; Chilkoti, A. Anal. Chem. 2002, 74, 504-509.

(79) Klabunde, K. J. In Nanoscale Materials in Chemistry; Paul, M., Ed.; John Wiley \& Sons Inc.: New York, 2001; p 129. are sufficient and effective to gauge the degree of the placeexchange reaction.

Improvement of the Sensitivity by Minimizing the Interparticular Repulsion. Experimental parameters associated with electrostatics on GNPs are intercorrelated and thus complicate the rationale. To straightforwardly demonstrate that the interparticle repulsion is a general and significant factor for the analytical performance, the experimental conditions should be constrained. Specifically, among the solution $\mathrm{pH}$, the ionic strength, and the amount of carboxylate on $15 \mathrm{c} 5-\mathrm{C}_{4}$ - $/ \mathrm{TA}-\mathrm{GNPs}$, two of them should be fixed to unambiguously examine how the repulsion dictated by the third factor affects the sensing performance. During the preparation of this manuscript, we thoroughly assessed a range of combination of these parameters and deduced a general guideline for the optimization of the sensing conditions. To better present the findings in the following, while the observation was made to show how GNP performance was a function of a particular factor, the other two were actually predetermined at their optimized conditions.

Effect of Solution $\mathbf{p H}$ on LOD. Figure 2A summarizes the effect of solution acidity on the interparticular repulsion, viz., the stability of $15 \mathrm{c} 5-\mathrm{C}_{4}$-/TA-GNPs. The data were derived from UV-vis spectra measured $1 \mathrm{~h}$ after the GNPs were exposed to the solution $\mathrm{pH}$ indicated in the abscissa. The ratio of $\epsilon_{525 \mathrm{~nm}} / \epsilon_{6600 \mathrm{~nm}}$ has been utilized as the flocculation parameter ${ }^{21}$ of gold sols because the numerator and the denominator evaluate the degree of dispersion and aggregation, respectively. A smaller $\epsilon_{525 \mathrm{~mm}} / \epsilon_{660 \mathrm{~mm}}$ indicates that the gold sols are less dispersive. When the solution acidity was pH 5.50 or more acidic, $0.70 \times$ GNPs began to exhibit flocculation in $\sim 1 \mathrm{~h}$ or less. A decrease in the TA coverage speeded up the flocculation at low solution $\mathrm{pH}$ and required a stronger solution basicity to keep the gold sols dispersive.

The response of GNPs after being exposed to $\mathrm{K}^{+}$is illustrated in Figure 2B. Figure 2C,D summarizes the effect of solution $\mathrm{pH}$ on the sensing performance of $21.4 \times$ GNPs. To make a conclusive comparison, all the solutions had a nominal ionic strength of 8.14 $\mathrm{mM}$ (see the following paragraph and the Supporting Information for the details). The best LOD of $21.4 \times \mathrm{GNPs}$ toward $\mathrm{K}^{+}$-sensing was found at $\mathrm{pH} 6.25$, the strongest acidity that $21.4 \times$ GNPs remained robustly stable. For solutions of $\mathrm{pH} 6.00$ or more acidic, 

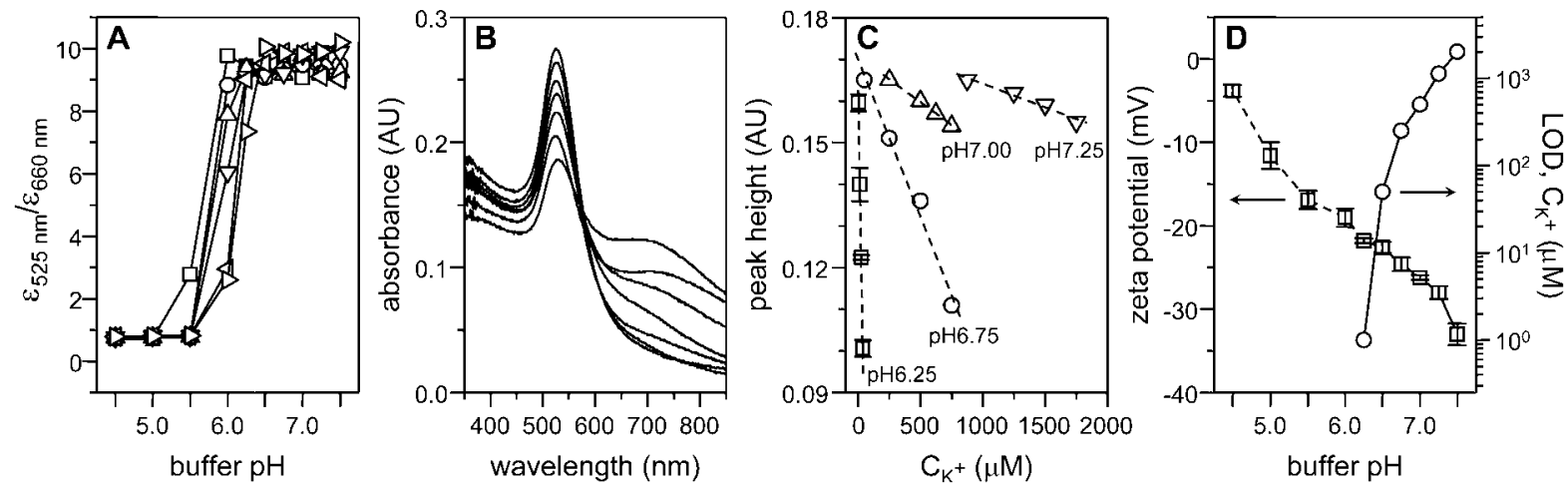

Figure 2. The effect of the solution $\mathrm{pH}$ on the analytical performance of $15 \mathrm{c} 5-\mathrm{C}_{4}-/ \mathrm{TA}-\mathrm{GNPs}$. (A) Flocculation parameter of $\epsilon_{525} \mathrm{~nm} / \epsilon_{660 \mathrm{~nm}}$ of the GNPs as a function of solution pH. Symbols: GNPs of $0.70 \times(\square), 3.6 \times(\bigcirc), 7.1 \times(\triangle), 14.3 \times(\nabla), 21.4 \times$ (left facing triangle), and $28.6 \times($ right facing triangle). (B) Spectroscopic response of $21.4 \times$ GNPs toward an aliquot of $0.1 \mathrm{~mL}$ of $\mathrm{K}^{+}$at pH 6.25. The curves from topmost to bottom corresponded to progressively increasing concentrations of $\mathrm{K}^{+}: 0.0,5.0,12.5,25.0,37.5,50.0$, and $75.0 \mu \mathrm{M}$. (C) The effect of the solution pH on the analytical performance of $21.4 \times$ GNPs. (D) $\zeta(\square)$ and LOD $(O)$ of $\mathrm{K}^{+}$-sensing for $21.4 \times$ GNPs against solution pH. UV-vis spectra and $\xi$ potentials were, respectively, acquired $1 \mathrm{~h}$ and $10-15 \mathrm{~min}$ after introducing $0.10 \mathrm{~mL}$ of GNPs into $1.80 \mathrm{~mL}$ solutions buffered to the reported $\mathrm{pH}$. The dashed trace in part D indicates where the $21.4 \times$ GNPs were flocculated and, therefore, the corresponding LODs were unavailable. Solution conditions: $10.0 \mathrm{mM}$ MES with an ionic strength of $8.14 \mathrm{mM}$.

the flocculation parameters, $\epsilon_{525 \mathrm{~mm}} / \epsilon_{660 \mathrm{~nm}}$, were small and indicative of unstable gold sols. The instability was attributed to the lack of sufficient repulsive charges as evidenced by the $\xi$ potential at the left ordinate of Figure 2D. The right ordinate of Figure 2D shows that as the solution acidity increased (i.e., a smaller $\mathrm{pH}$ ), $|\xi|$ of $21.4 \times$ GNPs decreased and the LOD concomitantly became better, demonstrating a strong correlation between the electrostatic repulsion and the sensing performance of GNPs. In other words, to achieve the optimal sensitivity, the solution $\mathrm{pH}$ should be determined at the strongest acidity that GNPs are stable yet have minimal electrostatic charges which render a small barrier for the recognition events to overcome. Noted that even under such conditions, the gold sols have sufficient selectivity to determine $\left[\mathrm{K}^{+}\right]$in solutions containing interferants and for human urine samples (vide infra).

Effect of Ionic Strength on LOD. $\zeta$ potential measurements (Figure S4 of Supporting Information) confirmed that a smaller $|\zeta|$ was found for $15 \mathrm{c} 5-\mathrm{C}_{4}-/ \mathrm{TA}-\mathrm{GNPs}$ in solutions of higher ionic strengths which developed a thinner Debye screening length and a smaller electrostatic barrier for the recognition event. Figure $3 \mathrm{~A}$ displays the sensitivity of $21.4 \times$ GNPs toward $\mathrm{K}^{+}$-sensing at ionic strengths of $6.14,7.14$, and $8.14 \mathrm{mM}$. The slopes are similar, suggesting an insignificant effect of the ionic strength on the detection sensitivity. For solutions with the ionic strength of 9.14 $\mathrm{mM}$ or more, the gold sols gradually flocculated within $1 \mathrm{~h}$ ( $\square$ and the dotted part in Figure 3B), and consequently experiments that examined the sensitivity were not carried out. The right ordinate of Figure 3B shows that increasing the ionic strength improves the LOD by orders of magnitude and that, at a fixed solution $\mathrm{pH}$, the best LOD can be obtained at the strongest ionic strength available.

Effect of the Amount of Functional Groups on LOD. XPS measurements found $47-92 \%$ coverage of $15 \mathrm{c} 5-\mathrm{C}_{4}-\mathrm{SH}$ for GNPs prepared by the place-exchange reaction. For each type of the GNPs, the corresponding optimal conditions were applied to determine the LODs, which would otherwise exhibit a difference by orders of magnitude as what presented in Figures 2D and 3B.
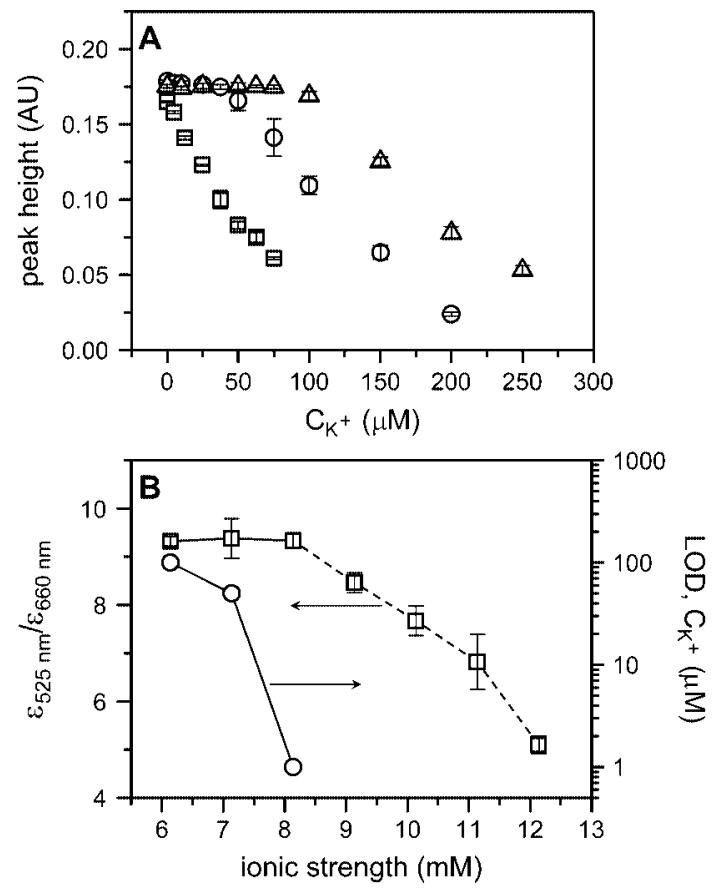

Figure 3. The effect of the ionic strength on $(A)$ the sensitivity and (B) flocculation parameter and LOD of $15 \mathrm{c} 5-\mathrm{C}_{4}-/$ TA-GNPs. UV - vis spectra for the assessment of the sensitivity and stability $\left(\epsilon_{525 \mathrm{~nm}} / \epsilon_{660 \mathrm{~nm}}\right)$ were measured, respectively, $3 \mathrm{~min}$ and $1 \mathrm{~h}$ after the preparation of the test solutions. Symbols in panel A: the ionic strength of $6.14(\triangle)$, $7.14(\bigcirc)$, and $8.14 \mathrm{mM}(\square)$. The dashed trace in panel B indicates under what $\mathrm{pH}$ the $21.4 \times \mathrm{GNPs}$ were flocculated. Solution conditions: $21.4 \times$ GNPs in 10.0 mM MES (pH 6.25).

Figure 4A displays the peak height of the GNPs as a function of $C_{\mathrm{K}^{+}}$at conditions of the highest ionic strength and strongest acidity yet truly stable for the GNPs. The corresponding LODs are summarized in Figure 4B and Figure S5 in Supporting Information.

The LOD was presumably improved with an increasing number of the sensing ligand on GNPs. ${ }^{8}$ The results in Figure $4 \mathrm{~B}$ generally followed this trend until the amount of crown ether was greater than that of $21.4 \times$ GNPs. The LODs of two additional compositions, $18.6 \times$ and $25.7 \times$ GNPs, were therefore prepared and were both found somewhat inferior to that of $21.4 \times$ GNPs. This 

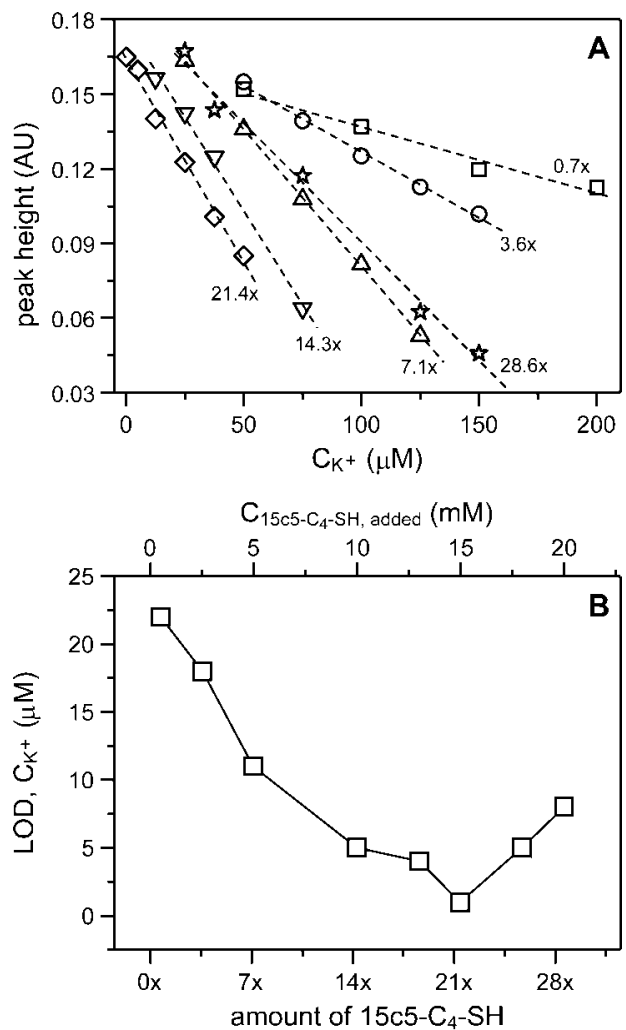

Figure 4. The effect of the amount of crown ether at GNPs on the LOD of $\mathrm{K}^{+}$-sensing. The notation in the abscissa indicates how the GNPs were prepared (see Experimental Section). Conditions: absorbance of $0.275 \mathrm{AU}$ at $\lambda_{525 \mathrm{~nm}}$ for GNPs; spectra acquired $3 \mathrm{~min}$ after introducing a $0.100 \mathrm{~mL}$ aliquot of the sample into $1.90 \mathrm{~mL}$ of GNPs; 10.0 mM MES; solution $\mathrm{pH}$, at the lowest $\mathrm{pH}$ where the GNPs were stable, $\mathrm{pH} 6.00$ for $0.70 \times$ and $3.6 \times$, $\mathrm{pH} 6.25$ for $7.1 \times, 14.3 \times$, $18.6 \times$, and $21.4 \times, \mathrm{pH} 6.50$ for $25.7 \times$ and $28.6 \times$ GNPs; ionic strength, under the above $\mathrm{pH}$ where the strongest ionic strength was available for stable GNPs, $8.14 \mathrm{mM}$ for $0.70 \times, 3.6 \times, 18.6 \times, 21.4 \times, 25.7 \times$, and $28.6 \times$ GNPs and $9.14 \mathrm{mM}$ for $7.1 \times$ and $14.3 \times$ GNPs.

confirmed that $21.4 \times$ GNPs conferred the best LOD. The fact that $25.7 \times$ and $28.6 \times$ GNPs had higher coverages of crown moiety yet showed slightly inferior analytical performances to $21.4 \times$ GNPs suggests that the presence of TA at the solution-GNP interface had an effect on the sensing efficiency of $15 \mathrm{c}^{-} \mathrm{C}_{4}$-/TA-GNPs. This appears to echo to our previous finding ${ }^{80}$ that the rate constant of $\mathrm{K}^{+}$recognition by $15 \mathrm{c} 5-\mathrm{C}_{4}-/ \mathrm{TA}-\mathrm{GNPs}$ was more than 4 orders of magnitude faster than those of $15 \mathrm{c} 5-\mathrm{C}_{8}$-/TA-GNPs and $15 \mathrm{c} 5$ $\mathrm{C}_{12}$-/TA-GNPs. Such a chain-length dependence was attributed to a cooperative effect between crown ether and the carboxylate group at TA. ${ }^{80}$

The LOD of $21.4 \times$ GNPs had a $\sim 30$-fold improvement $(0.2 \mu \mathrm{M})$ from our previous study $(6.5 \mu \mathrm{M})$ when the comparison was made by using the earlier experimental protocol that UV-vis spectra were obtained $15-20 \mathrm{~min}$ after introducing a $0.70 \mathrm{~mL}$ aliquot of samples into $1.80 \mathrm{~mL}$ of GNPs. ${ }^{80}$ On the other hand, the LODs of the eight types of GNPs were approximately on the same order of magnitude. The small difference in LODs, in fact, manifested the foremost finding of this thorough examination. For recognition schemes relying on the disperse-to-aggregation transformation, the effect of increasing the density of the sensing moiety on GNPs

(80) A concentration of $16.5 \mathrm{nM} 6 \times$ GNPs or absorbance of $1.36 \mathrm{AU}$ at $\lambda_{\max }, \mathrm{pH}$ 5.85 , and $5.34 \mathrm{mM}$ ionic strength for both the sample and gold sols.

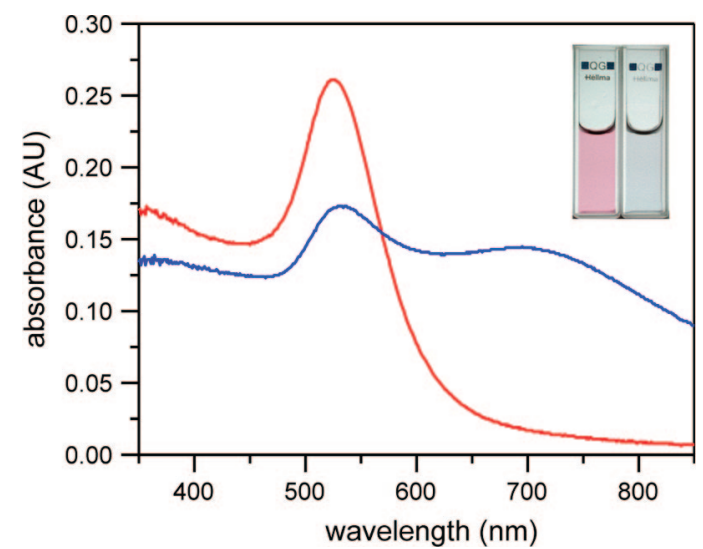

Figure 5. UV-visible spectra and photographs of solutions of $21.4 \times$ GNPs $(1.90 \mathrm{~mL}$ ) after subjected to addition of $0.10 \mathrm{~mL}$ of blank (red trace and the cuvette at the left) and of $0.10 \mathrm{~mL}$ of sample with additional $0.25 \mathrm{mM} \mathrm{K}^{+}$. Conditions: $10.0 \mathrm{mM} \mathrm{MES,} \mathrm{pH} 6.25$, ionic strength of $8.00 \mathrm{mM}$. The $0.10 \mathrm{~mL}$ of blank and sample both contained $\mathrm{Na}^{+}$(2.0 mM), $\mathrm{NH}_{4}^{+}(0.75 \mathrm{mM}), \mathrm{Mg}^{2+}(0.075 \mathrm{mM}), \mathrm{Ca}^{2+}(0.05 \mathrm{mM})$, $\mathrm{Zn}^{2+}(2.0 \mu \mathrm{M})$, and $\mathrm{Cu}^{2+}(0.050 \mu \mathrm{M})$ whose concentrations relative to that of $\mathrm{K}^{+}$were prepared based on ref 58 .

was not as significant as that of reducing the repulsion between GNPs. To be explicit, it was a very tedious and expensive task to determine the coverage of the sensing group on GNPs with the combining effects from of UV-vis, TEM, TGA, and XPS. It required numerous calibration curves to pin down the coverage that showed the best analytical performance. Even if the optimal coverage was a given, it was quite a challenge to modify GNPs exactly with that specific coverage. Figure $4 \mathrm{~B}$ demonstrated that these GNPs performed comparably under their optimal detection conditions. Therefore, to strive for the best sensing performance, minimizing the interparticular repulsion via tuning $\mathrm{pH}$ and ionic strength is a more efficient and controllable approach than optimizing the coverage of the sensing moiety on GNPs.

Real Sample Analysis: Selectivity of 15c5-C $\mathrm{C}_{4}$-/TA-GNPs under Conditions of Low Interparticular Repulsion. Figure 5 shows that, under the optimized $\mathrm{pH}$ and high ionic strength, $15 \mathrm{c}-\mathrm{C}_{4}$-/TA-GNPs selectively responded to $\mathrm{K}^{+}$in matrices containing interfering cations mimicking human urine samples. The red and blue traces were from $21.4 \times$ GNPs subjected to a $0.10 \mathrm{~mL}$ aliquot of, respectively, a blank solution and with additional $0.25 \mathrm{mM} \mathrm{K}$. Both the $0.10 \mathrm{~mL}$ aliquots contain physiologically important cations that were equivalent to 50 -fold dilution of real samples, viz., $\mathrm{Na}^{+}(2.0 \mathrm{mM}), \mathrm{NH}_{4}{ }^{+}(0.75 \mathrm{mM})$, $\mathrm{Mg}^{2+}(0.075 \mathrm{mM}), \mathrm{Ca}^{2+}(0.05 \mathrm{mM}), \mathrm{Zn}^{2+}(2.0 \mu \mathrm{M})$, and $\mathrm{Cu}^{2+}$ $(0.050 \mu \mathrm{M})$. The relative concentrations of $\mathrm{K}^{+}$to the other ions were prepared based on a literature report. ${ }^{58}$ The LODs for the GNPs were so low that the dilution of samples made the ionic strength impossible becoming higher than $8.14 \mathrm{mM} .^{58}$ The gold sols examined by the blank solution remained red (the left cuvette) while the other transformed into pale blue immediately upon being exposed to $\mathrm{K}^{+}$, demonstrating the excellent selectivity of $15 \mathrm{c} 5$ - $\mathrm{C}_{4}$-/TA-GNPs and the applicability of this approach toward $\mathrm{K}^{+}$sensing.

Real sample analysis was carried out to demonstrate that, even at low coverage of crown moiety, the approach of reducing the interparticular repulsion provided satisfactory results of $\mathrm{K}^{+}$ recognition. Following the previously reported protocol which had been interrogated by ICP-AES, ${ }^{38}$ the UV-vis spectra and calibra- 


\begin{tabular}{|c|c|c|c|}
\hline GNPs & $21.4 \times$ GNPs & $7.1 \times$ GNPs & arbitrary $\times$ GNPs \\
\hline linear equation & $y=0.164-1.642 x$ & $y=0.191-1.101 x$ & $y=0.188-0.807 x$ \\
\hline$r^{2}$ & 0.992 & 0.9998 & 0.995 \\
\hline $\begin{array}{l}\text { measured concentration } \\
(\mathrm{mM}) \text { for urine after dilution }\end{array}$ & $0.28 \pm 0.03$ (50-fold dilution) & $0.72 \pm 0.04$ (20-fold dilution) & $1.39 \pm 0.07$ (10-fold dilution) \\
\hline spike (mM, $n \geq 6)$ & 0.500 & 1.000 & 1.00 \\
\hline $\begin{array}{l}\text { concentration after spike } \\
\quad(\mathrm{mM}, n \geq 6)\end{array}$ & $0.79 \pm 0.04$ & $1.69 \pm 0.07$ & $2.38 \pm 0.05$ \\
\hline recovery $(\%)(n \geq 7)$ & 103 & 97 & 99 \\
\hline $\begin{array}{l}\text { concentration }(\mathrm{mM}) \text { in urine } \\
\text { determined by this method }(n \geq 6)\end{array}$ & $14.2 \pm 1.4$ & $14.4 \pm 0.8$ & $13.9 \pm 0.7$ \\
\hline
\end{tabular}

tion curves were obtained $15-20$ min after introducing the samples into the solutions of $21.4 \times$ GNPs. The calibration curve exhibited good linearity (for details, see Table 2 and Figure S6 in Supporting Information). The urine samples, whose concentration of $\mathrm{K}^{+}$typically falls in the range $25-125 \mathrm{mmol} / 24 \mathrm{~h},{ }^{58}$ were treated simply by dilution to minimize the matrix effect. The concentration of $\mathrm{K}^{+}$was found to be $14.2 \pm 1.4 \mathrm{mM}(n=6)$ and the recovery was $103 \%(n=7)$. A similar $\mathrm{K}^{+}$level and a spike recovery were measured by $7.1 \times$ GNPs. Also conducted was GNPs modified by an arbitrary amount of crown thiol. The measured $\left[\mathrm{K}^{+}\right]$was in good agreement with the other two GNPs although the relatively small slope of the calibration curve suggested a smaller coverage than that of $7.1 \times$ GNPs.

\section{CONCLUSIONS}

By taking advantage of the high extinction coefficients of GNPs, successful demonstration for the pretreatment of the complicated urine samples was simple dilution which made the matrix effect negligible. This can be achieved by either increasing the density of the sensing moiety on GNPs or by minimizing the repulsion between particles. The coverage of crown ether on GNPs was adjusted by place-exchange reactions and reached a limiting value which was dependent on the ratio of the number of the incoming crown ether to that of thioctic acid already on GNPs. With 4-day modification, the coverage of crown ether to the total thiol legs was found in the range of $47-92 \%$. An exhaustive examination of eight types of $15 \mathrm{c} 5-\mathrm{C}_{4}$-/TA-GNPs revealed that the sensitivity and LODs were a function of the coverage of crown ether moiety and that the one with a $87 \%$ exchange ratio (namely, 21.4× GNPs) exhibited the best analytical performance. Under the optimal conditions for each type of $15 \mathrm{c} 5-\mathrm{C}_{4}$-/TA-GNPs, their LODs were all sufficient for real sample analysis and actually differed only by 20 -fold. In other words, satisfactory LODs could also be obtained by minimizing the electrostatic repulsion between GNPs. From the practical aspect of condition optimization, adjusting solution $\mathrm{pH}$ and ionic strength were more controllable and efficient than tuning and determining the coverage of the sensing moiety. Therefore, when developing a GNP sensor, the general guideline for the improvement in LODs should be focused on reducing the repulsive energy barrier for the recognition event.

\section{ACKNOWLEDGMENT}

S.-H.W. and Y.-S.W. contributed equally to this work. The authors thank National Tsing Hua University and National Taiwan University for the strong research support. We are indebt to Dr. S.-Y. Lin for the synthesis of crown thiol, to Dr. I-W. P. Chen for the XPS measurements, to Dr. Ming-Chang Liu for TGA measurements, and to Prof. C.-Y. Mou (NTU, Department of Chemistry) and Trekintal Corporation for access to the $\zeta$ potential analyzers. C.-h.C. acknowledges the College of Science (NTU) for a startup fund (Grant 95R0034-02). This work was supported by National Science Council of ROC through Grants NSC-95-2113-M-002-036.

\section{SUPPORTING INFORMATION AVAILABLE}

Experimental details; IR spectra of $15 \mathrm{c} 5-\mathrm{C}_{4}-/ \mathrm{TA}-\mathrm{GNPs}$; calibration curves of GNPs concentrations against absorbance; the TGA diagram of TA-GNPs; the effect of the ionic strength on the $\zeta$ potential; UV-vis spectra and LODs for the GNPs; and calibration curves for real sample analysis. This material is available free of charge via the Internet at http://pubs.acs.org.

Received for review March 21, 2008. Accepted June 4, 2008.

\section{AC800589S}

